\title{
UM MODELO PARA A PROGRAMAÇÃO DE ROTAÇÕES DE CULTURAS
}

\author{
Lana Mara Rodrigues dos Santos \\ Departamento de Matemática \\ Universidade Federal de Viçosa (UFV) \\ Viçosa - MG \\ Ricardo Henrique Santos \\ Departamento de Fitotecnia \\ Universidade Federal de Viçosa (UFV) \\ Viçosa - MG \\ Marcos Nereu Arenales * \\ Dep. de Matemática Aplicada e Estatística / ICMC \\ Universidade de São Paulo (USP) \\ São Carlos - SP \\ arenales@icmc.usp.br \\ Luiz Aurélio Raggi \\ Departamento de Informática \\ Universidade Federal de Viçosa (UFV) \\ Viçosa - MG \\ * Corresponding author/autor para quem as correspondências devem ser encaminhadas \\ Recebido em 12/2006; aceito em 07/2007 \\ Received December 2006; accepted July 2007
}

\section{Resumo}

Neste artigo é apresentado um modelo de otimização 0-1 para determinar uma programação de rotações de culturas em uma área de plantio dividida em lotes. As culturas podem apresentar ciclos produtivos com durações distintas e variadas épocas do ano para o plantio. O modelo inclui restrições de plantio para lotes vizinhos e para seqüência de culturas na rotação, além do plantio de culturas para adubação verde e períodos de pousio. As rotações têm a mesma duração em todos os lotes e as culturas são selecionadas para maximizar a ocupação dos lotes. Foram realizados experimentos computacionais usando exemplos retirados de situações reais com rotações de 2 anos, envolvendo 28 culturas.

Palavras-chave: otimização linear inteira; rotação de culturas.

\begin{abstract}
This paper addresses the planning programming of crop rotation in neighbor plots. The crops can have productive cycles with different duration and varied seasons for the planting. The planning includes restrictions of planting for neighbor plots and for sequence of crops in the rotation. Moreover, there are included crops for green fertilizing and periods of fallow. The rotations have the same duration in each plot. A 0-1 linear programming problem was developed to deal with the problem of maximizing the allocation on the plots, subject to the conditions described before. Computational experiments were carried out using examples from the real world with rotations of 2 years and 28 cultures.
\end{abstract}

Keywords: integer linear programming; crop rotation. 


\section{Introdução}

Um dos eixos centrais dos modelos sustentáveis de produção vegetal é a diversificação das atividades nas áreas de cultivo para incrementar a sustentabilidade ecológica, econômica e social da agricultura. Estudos agronômicos e ecológicos demonstram que o aumento da diversidade de espécies vegetais no ambiente traz benefícios, tais como, a melhor exploração dos recursos produtivos, um menor ataque de herbívoros e menor incidência de patógenos, um maior controle de plantas espontâneas, maior produção por área e maior estabilidade da produção frente às pressões ambientais (Altieri, 2002; Gliessman, 2000; Vandermeer, 1989).

Dentre os critérios ecológicos, econômicos e agronômicos para o planejamento da diversificação de culturas, podem ser relacionados: requerimentos diferenciais de nutrientes, capacidade de cobertura do solo, expectativas de preços de comercialização da produção e lucratividade. No entanto, uma das principais finalidades do estabelecimento de áreas/ períodos com diferentes culturas é reduzir os recursos disponíveis tanto para artrópodes quanto microorganismos prejudiciais às lavouras, reduzindo assim suas populações e seus danos. Embora existam organismos-peste generalistas, muitos deles obtêm recursos de plantas da mesma família botânica. Assim, em muitos modelos sustentáveis de produção, a diversificação de culturas baseia-se na combinação de culturas de diferentes famílias botânicas no tempo e no espaço. Além disso, procura-se incluir nessas combinações espécies leguminosas, para a adição de nitrogênio, e períodos de pousio, em que a vegetação espontânea é deixada crescer livremente por um período definido, para a recuperação da estrutura e fauna do solo (Altieri, 2002; Gliessan, 2000).

A alocação de culturas no tempo e no espaço também deve atender às interações entre suas características fisiológicas e o clima local. Assim, mesmo em regiões tropicais nas quais é possível cultivar-se o ano inteiro (desde que haja irrigação disponível), existem culturas cujo desempenho é extremamente sazonal, com grandes restrições de época de cultivo.

Os produtores de hortaliças estão acostumados a lidar com a produção continuada de grande número de culturas, alocadas simultaneamente em áreas adjacentes. Contudo, essa forma de diversificação nem sempre segue um planejamento que otimize as vantagens desta forma de cultivo. Por outro lado, a produção dessas culturas é bastante complexa se comparada à produção de espécies florestais perenes ou culturas anuais pois compreendem um número grande de famílias botânicas, as culturas apresentam produtividades altíssimas e variáveis, os ciclos (períodos de cultivo) das culturas são curtos, embora com grande variabilidade, e apresentam limitações específicas quanto à época de cultivo. Portanto, no planejamento do plantio orgânico de hortaliças é necessário considerar, além dos critérios importantes para a diversificação ambiental listados anteriormente, os diferentes ciclos produtivos e exigências climáticas das culturas.

Algumas classes de problemas de rotação de culturas têm sido abordadas por meio de otimização linear por autores como Hildreth \& Reiter (1949), El-Nazer \& McCarl (1986), Clarke (1989), Doggliotti et al. (2003, 2004), Detlefsen \& Jensen (2007), Haneveld \& Stegeman (2005).

Nos modelos desenvolvidos por El-Nazer \& McCarl (1986), Detlefsen \& Jensen (2007) e Haneveld \& Stegeman (2005), as variáveis de decisão são áreas onde certas seqüências admissíveis de culturas são alocadas. Nestes modelos não há restrições de plantio para áreas adjacentes e existe única seção de plantio anual. No modelo linear em Clarke (1989), as culturas podem apresentar diferentes ciclos de cultivo e época de plantio. No entanto, não existem restrições de plantio seqüencial ou para áreas vizinhas. 
Neste artigo é proposto e analisado um modelo de otimização 0-1 para rotação de culturas em diversas áreas, voltado para a produção agroecológica de hortaliças. O objetivo é maximizar a ocupação das áreas de plantio sujeito a restrições de vizinhança e sucessão para culturas de mesma família botânica, além do plantio de uma cultura para adubação verde e inclusão de um período de pousio em cada rotação.

$\mathrm{Na}$ próxima seção são introduzidos alguns conceitos relacionados à rotação de culturas. $\mathrm{Na}$ Seção 3, um modelo para a programação de rotações de culturas em um conjunto de lotes é apresentado assim como resultados computacionais e restrições adicionais que podem ser incorporadas ao modelo. Na Seção 4 é proposta uma subdivisão particular do problema em subproblemas menores que, em alguns casos, garante a otimalidade do problema original. $\mathrm{Na}$ Seção 5 apresentamos algumas conclusões e perspectivas futuras.

\section{Programação de rotações de culturas}

A rotação de culturas é um sistema de plantio seqüencial de espécies vegetais sobre a mesma área, de modo que esta seqüência se repita a partir de um determinado período de tempo. Em particular, dadas as culturas 1 e 2, a rotação 1-2, indica que após o cultivo da cultura 1, segue o da cultura 2; depois da 2, segue o da cultura 1; e assim sucessivamente.

O tamanho de uma rotação é o intervalo de tempo necessário para que tenha início a repetição da seqüência de culturas. No modelo matemático apresentado na Seção 3, as rotações têm o mesmo tamanho em todos os lotes. Esta condição é imposta para tratar das restrições de plantio em áreas vizinhas.

A programação de uma rotação é um calendário de plantio que exibe o período do plantio à colheita das culturas selecionadas para cada rotação. Por exemplo, sejam as culturas 1-5, com ciclos de plantio respectivamente 4, 2, 2, 2, 3 e 1. A Figura 1.1 ilustra uma programação de 6 períodos em duas áreas (isto é, cada rotação é de tamanho 6), com as 5 culturas: a rotação 5-3-4 na área 1, e a rotação 2-1 na área 2. Em particular, a cultura 1 é cultivada na área 2 nos períodos $5,6,1$ e 2 .

\begin{tabular}{c|c|c|c|c|c|c|}
\hline Área 2 & & & 2 & & 1 & \\
\hline Área 1 & & & & 3 & & 4 \\
\hline Período & 1 & 2 & 3 & 4 & 5 & 6
\end{tabular}

Figura 1.1 - Programação para 2 áreas em 6 períodos.

\section{Um modelo para a programação de rotações}

Dado um conjunto de lotes para plantio, um tamanho de rotação (igual para todos os lotes) e um conjunto de culturas que podem ser selecionadas para as rotações, deseja-se encontrar uma programação de rotações que maximize a ocupação dos lotes. As rotações estão sujeitas às restrições de $(i) \mathrm{a}(v)$ :

(i) Restrições intrínsecas às culturas: família botânica a que pertencem, época de cultivo e ciclo de plantio (tempo de cultivo).

(ii) Restrições de vizinhança para culturas de mesma família: culturas de mesma família não são cultivadas em uma mesma época em lotes adjacentes. 
(iii) Restrições de continuidade para culturas de mesma família botânica: culturas de mesma família não são plantadas em um mesmo lote consecutivamente.

(iv) Alocação do adubo verde: as leguminosas selecionadas para adubação verde se sujeitam às condições (ii) e (iii) e, em cada lote, uma delas é cultivada uma vez.

(v) Alocação do período de pousio: em lote, programa-se um período de pousio.

\subsection{Formulação matemática}

Considera-se que o tamanho da programação $(T)$ é dividido em $M$ períodos de mesma duração e a área de plantio é dividida em $L$ lotes. Para um conjunto de $N$ culturas em $N F$ famílias botânicas, o modelo de programação de rotação de culturas $(P R C)$ pode ser expresso pelo modelo de otimização $0-1$ a seguir. Por conveniência, o período de pousio é representado pela cultura $n=N+1$.

$$
\max \sum_{k=1}^{L} \sum_{j \in I_{i}} \sum_{i \in C} t_{i} x_{i j k}
$$

Sujeito a :

$$
\begin{aligned}
& \sum_{i=1}^{N} \sum_{r=0}^{t_{i}-1} x_{i(j-r) k} \leq 1, j=1 . . M, k=1 . . L \\
& \sum_{i \in F p} \sum_{r=0}^{t_{i}-1} x_{i(j-r) k}+x_{i(j-r) k+1} \leq 1, j=1 . . M, p=1 . . N F, k=1 . . L-1 \\
& \sum_{i \in F p} \sum_{r=0}^{t_{i}} x_{i(j-r) k} \leq 1, p=1 . . N F, j=1 . . M, k=1 . . L \\
& \sum_{j=1}^{M} \sum_{i \in A} x_{i j k}=1, k=1 . . L \\
& \sum_{j=1}^{M} x_{n j k}=1, k=1 . . L \\
& x_{i j k} \in\{0,1\}, i=1 . . N, j \in I_{i}, k=1 . . L
\end{aligned}
$$

* Nas equações (3.2), (3.3) e (3.4), se $j-r \leq 0$, substitui-se $j-r$ por $j-r+M$.

Variável:

$x_{i j k}=1$, se a cultura $i$ tiver seu plantio iniciado no período $j$ no lote $k, 0$ caso contrário.

\section{Parâmetros:}

$C$ : conjunto de culturas que podem ser selecionadas para as rotações, excluindo as culturas para adubação verde;

$A$ : conjunto de culturas para adubação verde;

$F_{p}:$ conjunto de culturas da família botânica $p, p=1 . . N F$; 
$t_{i}$ : ciclo de plantio da cultura $i$, incluindo o período estimado para preparo do solo e colheita;

$I_{i}=\left[C_{i}, T_{i}\right]$ : intervalo em que a cultura $i$ pode iniciar seu plantio, em que $C_{i}$ é o período mais cedo de plantio e $T_{i}$ o período mais tarde.

A função objetivo (3.1) fornece o tempo total de cultivo, a ser maximizado, e permite que todas as culturas tenham possibilidade de serem escolhidas sem influência significativa de seu ciclo de cultivo. As variáveis relativas às culturas para a adubação verde foram excluídas da função objetivo, pois se exige apenas que, em cada rotação, uma delas seja cultivada uma única vez. O mesmo se aplica ao período de pousio.

As restrições (3.2) impedem que duas culturas ocupem o mesmo espaço. Isto é, se uma cultura é plantada em um lote, nenhuma outra cultura poderá ser plantada no mesmo lote, até o término do plantio daquela cultura. As restrições (3.3) proíbem que culturas $i$ e $j$ de mesma família sejam cultivadas em lotes adjacentes, coincidindo em algum período de tempo.

As restrições (3.4) garantem que culturas de mesma família não são plantadas consecutivamente em um mesmo lote. Não é necessário que estas restrições sejam estendidas às culturas para adubação verde ou período de pousio, pois as restrições (3.5) e (3.6) já garantem isso.

\subsection{Testes computacionais}

Para realização dos experimentos computacionais da formulação matemática apresentada na Seção 3.1, foi usado o aplicativo comercial CPLEX 7.5 (ILOG, 2001), versão para Windows, em um Pentium 4-2.8GHZ com 528MB de RAM. Foram selecionadas 28 culturas, 4 delas para adubação verde. Cada período foi fixado em 10 dias, o tamanho de rotação em 2 anos (72 períodos) e estabeleceu-se um período de pousio de um mês ( 3 períodos) para todos os lotes.

Fez-se uma programação de rotações de culturas para conjuntos de 1 a 10 lotes. Alguns resultados destes experimentos são exibidos na Tabela 1. O número de variáveis e restrições são os obtidos pelo CPLEX depois do pré-processamento.

Tabela 1 - Resultados computacionais obtidos com o CPLEX 7.5.

\begin{tabular}{c|c|c|c|c|c}
\hline \hline Número de lotes & Restrições & Variáveis & Tempo(seg) & Iterações & Nós \\
\hline \hline 1 & 520 & 983 & 0,20 & 228 & 0 \\
2 & 1947 & 2280 & 1,06 & 559 & 0 \\
3 & 3258 & 3420 & 212,66 & 43.474 & 60 \\
4 & 4573 & 4560 & 600,80 & 106.644 & 110 \\
5 & 5883 & 5700 & 325,49 & 22.944 & 0 \\
6 & 7195 & 6840 & $4.120,50$ & 421.239 & 330 \\
7 & 8509 & 7980 & $7.261,19$ & 797.113 & 345 \\
8 & 9818 & 9120 & $10.114,86$ & 786.871 & 411 \\
9 & 11068 & 10110 & $16.711,39$ & 1.272 .975 & 268 \\
10 & 12446 & 11400 & $29.498,23$ & 2.105 .110 & 718 \\
\hline \hline
\end{tabular}


Percebe-se que, com o aumento no número de lotes há um aumento considerável no número de restrições e de variáveis, o que acarreta um significativo aumento em outros valores, como o tempo computacional. Alternativas para minorar este problema são discutidas na Seção 4.

Os valores utilizados nos experimentos computacionais (número máximo de lotes, unidade de tempo, tamanho da rotação, época de plantio e ciclo das culturas), foram obtidos na literatura em Filgueira (2003), Santos (2005) e Souza \& Resende (2006), e na horta orgânica da Universidade Federal de Viçosa-MG. Os dados reais das 28 culturas para os experimentos computacionais, famílias a que pertencem, épocas de plantio e ciclos de plantio são apresentados na Tabela A.1 no Apêndice A. As culturas 25-28 são as selecionadas para adubação verde. O período obrigatório de pousio é representado pela cultura 29. A Figura 2 exibe um diagrama de alocação para 10 lotes obtidos nos testes computacionais.

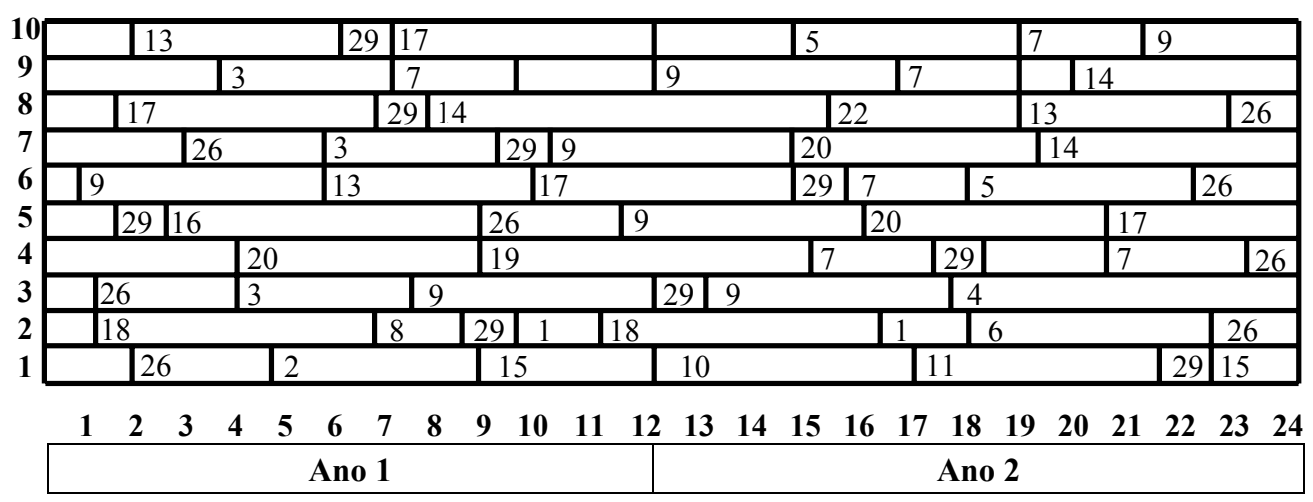

Figura 2 - Programação ótima para 10 lotes.

\subsection{Considerações adicionais}

Embora o problema em estudo neste artigo tenha como objetivo a alocação máxima da área de plantio, outros objetivos podem ser desejados, tais como, otimizar produtividade ou lucro. Além disto, em muitos problemas práticos envolvendo rotação de culturas restrições adicionais podem (ou devem) ser incorporadas ao modelo. Por exemplo, pode-se priorizar (ou mesmo obrigar) o cultivo de determinadas culturas ou interromper a programação de rotações e iniciá-la em um momento específico.

Restrições adicionais são incorporadas ao modelo $P R C$ com novas equações/inequações, com a alteração de limitantes em restrições existentes ou fixação em zero de um apropriado conjunto de variáveis. Em particular, para priorizar um conjunto de culturas, uma alternativa é alterar na função objetivo os coeficientes das variáveis associadas a elas. Em uma situação em que se deseje cultivar uma cultura $i$, com freqüência $l_{i}$, é suficiente acrescentar a restrição (3.8).

$$
\sum_{j \in I_{i}} \sum_{k=1}^{L} x_{i j k} \geq l_{i}
$$

É claro que, dependendo das restrições adicionais o problema pode não ter solução para o tamanho de rotação fixado. Neste caso, pode-se, por exemplo, mudar o tamanho da rotação 
e/ou relaxar, dentre outras, a restrição de vizinhança para culturas de uma mesma família. Para relaxar as restrições (3.3) parcialmente, permitindo que culturas de uma mesma família sejam cultivadas em lotes adjacentes coincidindo em, no máximo, $s$ unidades de tempo, substitui-se $t_{i}-1$ nas restrições (3.3) por $t_{i}-1-s$, obtendo-se assim as restrições (3.9).

$$
\begin{aligned}
\sum_{i \in F_{p}} \sum_{r=0}^{t_{i}-1-s} x_{i(j-r) k}+x_{i(j-r) k+1} \leq 1, j \in I_{i}, p=1 . . N F, k & =1 . . L-1 \\
& * \operatorname{Se} j-r \leq 0 \text { substitui-se } j-r \text { por } j-r+M .
\end{aligned}
$$

\section{Subconjuntos de lotes}

O número de restrições e variáveis do modelo pode ser muito grande e, para muitos exemplos gerados aleatoriamente, o CPLEX 7.5 não conseguiu garantir a solução ótima em um tempo computacional razoável. Para $N, M$ e $N F$ fixos (porque são valores que normalmente não se quer alterar), uma alternativa para diminuir o número de restrições e variáveis do modelo é subdividir o problema original em subproblemas com um menor número de lotes cada um. Uma programação para $R \leq L$ lotes pode ser obtida da seguinte maneira:

Considere uma partição do conjunto de L lotes em uma seqüência de K subconjuntos de lotes (de mesmo tamanho ou não), mantendo-se a ordem inicial dos lotes. Por exemplo, o conjunto de $L=5$ lotes pode ser particionado em $\{1,2,3\}$ e $\{4,5\}$.

Faz-se uma programação factível (ou ótima) para o primeiro desses subconjuntos. Para o primeiro lote do próximo subconjunto, são fixadas em zero as variáveis que garantem que as restrições (3.3) sejam satisfeitas, ou seja, com a alocação já realizada das culturas do último lote do subconjunto anterior, a alocação das culturas no primeiro lote do próximo subconjunto deve ser de modo que não viole as restrições relacionadas à vizinhança de culturas de mesma família.

Repete-se o procedimento para os subconjuntos de lotes seguintes até o fim da seqüencia ou não existir programação factível para o próximo subconjunto de lotes com esta restrição adicional.

Em particular, para $K=L$, este procedimento heurístico fornece bons resultados e rapidamente pois cada subconjunto tem apenas um lote e as restrições (3.3) são eliminadas completamente.

Uma solução ótima para o problema de programação de rotação de culturas $(P R C)$ pode ser obtida a partir de uma solução ótima para 2 lotes adjacentes. A demonstração desta afirmação encontra-se no Apêndice B.

É evidente que, ao dividir o conjunto de $L$ lotes e encontrar uma programação ótima para cada subconjunto de lotes ou repetir uma programação para outros subconjuntos de lotes, quando isto for possível, pode-se perder a otimalidade ou a diversidade de culturas obtida com a programação sem esta divisão. Para favorecer a diversificação, podem ser introduzidas alterações nos coeficientes da função objetivo do modelo, priorizando a alocação de um determinado conjunto de culturas. Neste caso, uma estratégia é modificar os coeficientes das variáveis relativas às culturas que não foram ainda alocadas ou penalizar as variáveis associadas às culturas que foram alocadas nos subproblemas anteriores. Essa penalização pode ser proporcional ao número de vezes que a cultura já foi alocada. 


\section{Exemplo ilustrativo}

Para este exemplo ilustrativo, a programação das rotações nos 10 lotes foi feita de duas diferentes maneiras, nomeadas de estratégia 1 e 2. Nas duas, o conjunto de 10 lotes foi dividido em 5 subconjuntos de 2. O aplicativo CPLEX 7.5 foi utilizado para a obtenção dos resultados.

Na estratégia 1, preenchido otimamente os 2 primeiros lotes (problema 1.1), são fixadas em zero as variáveis associadas ao terceiro lote que garantem desta forma que as restrições (3.3), sejam satisfeitas. O problema 1.2 refere-se à programação dos próximos dois lotes. Neste caso, foram penalizados (multiplicados por $1 / 2$ ), na função objetivo, os coeficientes associados às culturas já alocadas nos primeiros 2 lotes. Analogamente, foram construídos os problemas $1.3,1.4$ e 1.5 . Em cada um deles foram penalizados (multiplicados por $1 / 2$ ) na função objetivo os coeficientes associados às culturas já alocadas nos subconjuntos anteriores.

Na estratégia 2 foram gerados os problemas 2.1 a 2.5 , de maneira análoga à estratégia 1 . Porém, neste caso, não foram alteradas as funções objetivos dos subproblemas.

Com a divisão do conjunto de 10 lotes em 5 subconjuntos de 2 lotes, o número de restrições e de variáveis obtido pela soma do conjunto de restrições e de variáveis dos subproblemas nas estratégias 1 e 2, são ligeiramente inferiores ao total obtido no modelo $P R C$ sem a subdivisão do conjunto de lote. No entanto, o tempo computacional é bem inferior se comparado com a resolução do modelo sem a divisão do conjunto de lotes. Além disto, obteve-se alocação ótima e uma maior diversidade de culturas: 22, 20 e 18, respectivamente, para as estratégias 1 e 2, e para o modelo padrão. Os resultados computacionais, para os modelos (com os respectivos subproblemas), podem ser vistos na Tabela 2. O número de variáveis e de restrições são os obtidos pelo CPLEX depois do pré-processamento. A última linha da Tabela 2 exibe, em seqüência: o número de restrições, de variáveis, o tempo computacional e o número de culturas distintas obtidos com o modelo sem a subdivisão do conjunto de lotes.

Tabela 2 - Resultados computacionais.

\begin{tabular}{c|c|c|c|c|c}
\hline \hline Estratégia & $\begin{array}{c}\text { Subconjuntos } \\
\text { de Lotes }\end{array}$ & Restrições & Variáveis & $\begin{array}{c}\text { Tempo } \\
\text { (seg) }\end{array}$ & $\begin{array}{c}\text { Culturas } \\
\text { Distintas }\end{array}$ \\
\hline \hline & 1.1 & 1947 & 2280 & 1,06 & \\
\multirow{2}{*}{1} & 1.2 & 1820 & 1908 & 7,73 & \\
& 1.3 & 1812 & 2002 & 0,95 & \\
& 1.4 & 1800 & 1943 & 8,88 & \\
& 1.5 & 1854 & 1994 & 13,25 & \\
& $\mathbf{1 0}(\mathbf{1})$ & $\mathbf{9 . 2 3 3}$ & $\mathbf{1 0 . 1 2 7}$ & $\mathbf{3 1 , 8 7}$ & $\mathbf{2 2}$ \\
\hline & 2.1 & 1947 & 2280 & 1,06 & \\
& 2.2 & 1823 & 1908 & 11,20 & \\
& 2.3 & 1806 & 1944 & 12,01 & \\
& 2.4 & 1820 & 1915 & 6,55 & \\
& 2.5 & 1840 & 1931 & 0,86 & $\mathbf{2 0}$ \\
\hline \hline & $\mathbf{1 0}(\mathbf{2})$ & $\mathbf{9 . 2 3 6}$ & $\mathbf{9 . 9 7 8}$ & $\mathbf{3 1 , 6 8}$ & $\mathbf{1 8}$ \\
\hline \hline
\end{tabular}


Como foi mencionado na Seção 3.3, a inserção de restrições adicionais expressa situações freqüentes em problemas reais. No entanto, com divisão do conjunto de lotes em subconjuntos menores, a inserção de restrições adicionais pode exigir restrições diferentes para cada subproblema e isto resultar em uma programação distante da ótima ou mesmo infactível. A busca por estratégias de preenchimento para os subproblemas que forneçam soluções mesmo que somente factíveis, pode exigir um esforço computacional de mesma grandeza que o exigido para a programação sem a divisão dos lotes.

De qualquer forma, se o problema exige restrições adicionais, a programação obtida com a divisão do conjunto de lotes, pode ser útil para fornecer limitantes superiores ou programações factíveis: com o problema relaxado tem-se um limitante superior, e ao incorporar primeiro as restrições adicionais ao modelo e depois resolvê-lo com a divisão do conjunto de lotes, para uma dada estratégia obtém-se soluções factíveis.

\section{Conclusões}

Neste trabalho, a otimização matemática foi usada na solução do problema programação de rotações de culturas como uma ferramenta capaz de auxiliar o agricultor na tomada de decisão. As perspectivas de sua aplicabilidade são reais. Uma vez plenamente desenvolvida e com interface de fácil manuseio pelos usuários, a metodologia poderia ser empregada em propriedades agrícolas voltadas para a agricultura orgânica e, ainda, utilizada por centros de pesquisa agropecuária que poderiam gerar modelos de ocupação da área a serem difundidos para produtores de regiões de mesmas características climáticas.

Um modelo de programação 0-1 para determinar uma programação de rotações de culturas para um conjunto de lotes adjacentes, de modo a maximizar a alocação nos lotes nos tempos foi apresentado na Seção 3. As culturas podem apresentar ciclos produtivos com duração distinta e variadas épocas do ano para o plantio e cultivo. O modelo inclui, além de tais recomendações técnicas, restrições de vizinhança e sucessões para culturas de mesma família; plantio de culturas para adubação verde e um período de pousio em cada lote. As rotações têm a mesma duração em todos os lotes e as culturas na rotação são escolhidas de uma lista de culturas. Foram realizados experimentos computacionais usando exemplos retirados de situações reais, com rotações de 2 anos envolvendo 28 culturas. Todos os resultados computacionais foram obtidos usando o CPLEX 7.5.

A dificuldade de solução, em razão do porte que o modelo $P R C$ assume, pode ser contornada, por exemplo, com a subdivisão do conjunto de lotes em subconjuntos menores. O exemplo apresentado na Seção 4.1 ilustrou esta possibilidade. Não havendo restrições adicionais, uma solução ótima é encontrada com a divisão do conjunto de lotes em conjunto de 2 lotes. Dependendo do conjunto de restrições adicionais esta estratégia pode ser usada para encontrar limitantes.

\section{Agradecimentos}

Os autores agradecem aos revisores anônimos da revista Pesquisa Operacional, cujas sugestões melhoraram a qualidade final do artigo. Este trabalho teve apoio da CAPES, CNPq e FAPESP. 


\section{Referências Bibliográficas}

(1) Altieri, M.A. (2002). Agroecologia: bases cientificas para uma agricultura sustentável. Editora Agropecuária, Guaíba.

(2) Clarke, H.R. (1989). Combinatorial aspects of cropping pattern selection in agriculture. European Journal of Operational Research, 40, 70-77.

(3) Detlefsen, N. \& Jensen, A.L. (2007). Modelling optimal crop sequences using network flows. Agricultural Systems, 94, 566-572.

(4) Dogliotti, S.; Rossing, W.A.H. \& Van Ittersum, M.K. (2003). ROTAT, a tool for systematically generating crop rotations. European Journal of Agronomy, 19, 239-250.

(5) Dogliotti, S.; Rossing, W.A.H. \& Van Ittersum, M.K. (2004). Systematic design and evaluation of crop rotations enhancing soil conservation, soil fertility and farm income: a case study for vegetable farms in South Uruguay. Agricultural Systems, 80, 277-302.

(6) El-Nazer, T. \& McCarl, B.A. (1986). The choice of crop rotation: A modeling approach and case study. American Journal of Agricultural Economics, 68(1), 127-136.

(7) Filgueira, F.A.R. (2003). Novo Manual de Olericultura. Ed. UFV, Viçosa.

(8) Gliessman, S.R. (2000). Agroecologia: processos ecológicos em agricultura sustentável. Editora da Universidade - UFRGS, Porto Alegre.

(9) Haneveld, W.K. \& Stegeman, A.W. (2005). Crop succession requirements in agricultural production planning. European Journal of Operations Research, 166, 406-429.

(10) Hildreth, C.G. \& Reiter, S. (1949). On the choice of a crop rotation plan. In: Proceedings of the Conference on Linear Programming held in Chicago [edited by T.C. Koopmans], 177-188.

(11) ILOG (2001). ILOG CPLEX 7.5 Reference Manual. 7.5v. Copyright by ILOG, France.

(12) Santos, R.H.S. (2005). Olericultura orgânica. Cap. 18. In: Olericultura: teoria e prática [edited by P.C.R. Fontes], UFV, Viçosa, 249-276.

(13) Souza, J.L. de \& Resende, P.L. (2006). Manual de Horticultura Orgânica. Aprenda Fácil, Viçosa.

(14) Vandermeer, J.H. (1989). The Ecology of Intercropping. Cambridge University Press. 


\section{Apêndice A}

A Tabela A.1 exibe 28 culturas selecionadas para os experimentos, famílias às quais pertencem, épocas de plantio e ciclos de plantio. No ciclo de cada cultura está incluso o intervalo de tempo estimado para plantio e colheita.

Tabela A.1 - Dados sobre 28 culturas.

\begin{tabular}{|c|c|c|c|c|c|c|c|}
\hline & \multirow{2}{*}{ CULTURA } & \multirow{2}{*}{\multicolumn{2}{|c|}{ FAMÍLIA }} & \multicolumn{2}{|c|}{ ÉPOCA PLANTIO } & \multicolumn{2}{|c|}{ CICLO } \\
\hline & & & & Início & Fim & dias & $t$ \\
\hline 1 & Alface & 1 & Compositae & \multicolumn{2}{|c|}{ ano todo } & 50 & 5 \\
\hline 2 & Almeirão & 1 & Compositae & \multicolumn{2}{|c|}{ ano todo } & 120 & 12 \\
\hline 3 & Couve & 2 & Brassicaceae & março & junho & 100 & 10 \\
\hline 4 & Brócolis & 2 & Brassicaceae & fevereiro & junho & 230 & 23 \\
\hline 5 & Repolho & 2 & Brassicaceae & fevereiro & julho & 130 & 13 \\
\hline 6 & Couve-Flor & 2 & Brassicaceae & março & junho & 140 & 14 \\
\hline 7 & Beterraba & 3 & Chenopodiaceae & março & julho & 70 & 7 \\
\hline 8 & Espinafre & 3 & Chenopodiaceae & fevereiro & julho & 50 & 5 \\
\hline 9 & Abobrinha & 4 & Cucurbitaceae & agosto & março & 140 & 14 \\
\hline 10 & Moranga & 4 & Cucurbitaceae & setembro & janeiro & 150 & 15 \\
\hline 11 & Pepino & 4 & Cucurbitaceae & \multicolumn{2}{|c|}{ ano todo } & 140 & 14 \\
\hline 12 & Alho & 5 & Liliaceae & março & abril & 180 & 18 \\
\hline 13 & Cebola & 5 & Liliaceae & março & junho & 120 & 12 \\
\hline 14 & Quiabo & 6 & Malvaceae & agosto & março & 230 & 23 \\
\hline 15 & Milho & 7 & Gramineae & agosto & abril & 100 & 10 \\
\hline 16 & Aveia & 7 & Gramineae & março & maio & 180 & 18 \\
\hline 17 & Tomate & 8 & Solanaceae & \multicolumn{2}{|c|}{ ano todo } & 150 & 15 \\
\hline 18 & Pimentão & 8 & Solanaceae & \multicolumn{2}{|c|}{ ano todo } & 160 & 16 \\
\hline 19 & Jiló & 8 & Solanaceae & setembro & fevereiro & 190 & 19 \\
\hline 20 & Cenoura & 9 & Umbelliferae & março & julho & 140 & 14 \\
\hline 21 & Salsinha & 9 & Umbelliferae & setembro & março & 190 & 19 \\
\hline 22 & Feijão Vagem & 10 & Leguminosae & agosto & abril & 110 & 11 \\
\hline 23 & Ervilha & 10 & Leguminosae & março & abril & 90 & 9 \\
\hline 24 & Feijão & 10 & Leguminosae & agosto & setembro & 90 & 9 \\
\hline 25 & Crotalária & 10 & Leguminosae & setembro & dezembro & 90 & 9 \\
\hline 26 & Feijão-de-Porco & 10 & Leguminosae & setembro & dezembro & 80 & 8 \\
\hline 27 & Mucuna & 10 & Leguminosae & setembro & janeiro & 110 & 11 \\
\hline 28 & Ervilha Peluda & 10 & Leguminosae & março & junho & 140 & 14 \\
\hline
\end{tabular}

*Adaptado de Filgueira (2003); Santos (2005) e Souza \& Resende (2006). 


\section{Apêndice B}

Na Seção 4 foi descrito um procedimento para obtenção de uma solução heurística para o problema de programação de rotações de culturas $(P R C)$, baseado em subdivisão do conjunto de lotes. Neste apêndice é mostrado que uma solução ótima para o problema $P R C$ pode ser obtida com uma solução ótima para dois lotes adjacentes, conforme a proposição a seguir.

Proposição: Uma solução ótima do modelo de programações de rotações de culturas (PRC) para L lotes $(L>1)$ pode ser construída a partir de uma solução ótima para 2 lotes adjacentes.

Prova: por simplicidade, o modelo matemático descrito na Seção 3.1 pode ser reescrito na forma matricial abaixo.

$$
\begin{aligned}
& \max \quad T \mathrm{x}_{1}+\cdots+T \mathrm{x}_{L} \\
& \text { s.a. } P \mathrm{x}_{k}+P \mathrm{x}_{k+1} \leq \mathbf{1}, k=1 . . L-1 \\
& \quad \mathrm{x}_{k} \in S, \quad k=1 . . L
\end{aligned}
$$

em que $S$ é o conjunto de todas as rotações factíveis para um lote, $T$ o vetor de custos e $P$ a matriz associado às restrições (3.3).

Considere os problemas $P P$ e $P I$ como definidos abaixo.

$$
\begin{aligned}
& \max T \mathrm{x}_{1}+T \mathrm{x}_{2} \\
& \text { s.a. } P \mathrm{x}_{1}+P \mathrm{x}_{2} \leq \mathbf{1} \\
& \mathrm{x}_{1}, \mathrm{x}_{2} \in S \\
& \max \frac{L+1}{2} T \mathrm{x}_{1}+\frac{L-1}{2} T \mathrm{x}_{2} \\
& \text { s.a. } P \mathrm{x}_{1}+P \mathrm{x}_{2} \leq 1 \\
& \mathrm{x}_{1}, \mathrm{x}_{2} \in S
\end{aligned}
$$

Seja $L$ par e $\left(\tilde{\mathrm{x}}_{1}, \tilde{\mathrm{x}}_{2}\right)$ uma solução ótima de $P P$. A solução $\left(\tilde{\mathrm{x}}_{1}, \cdots, \tilde{\mathrm{x}}_{L}\right)$, em que $\tilde{\mathrm{x}}_{k}=\tilde{\mathrm{x}}_{1}$, para $k$ ímpar e $\tilde{\mathrm{x}}_{k}=\tilde{\mathrm{x}}_{2}$, para $k$ par é factível para o problema $P R C$ pois

$$
P \tilde{\mathrm{x}}_{k}+P \tilde{\mathrm{x}}_{k+1}=P \tilde{\mathrm{x}}_{1}+P \tilde{\mathrm{x}}_{2} \leq 1, k=1 . . L-1 .
$$

Para esta solução,

$$
T \tilde{\mathrm{x}}_{1}+\cdots+T \tilde{\mathrm{x}}_{L}=\frac{L}{2}\left[T \tilde{\mathrm{x}}_{1}+T \tilde{\mathrm{x}}_{2}\right]
$$

Seja $\left(\mathrm{x}_{1}, \cdots, \mathrm{x}_{L}\right)$ factível para o problema $P R C$. Como $P \mathrm{x}_{k}+P \mathrm{x}_{k+1} \leq \mathbf{1}$, então $\left(\mathrm{x}_{k}, \mathrm{x}_{k+1}\right)$, $k=1 . . L-1$ são factíveis para $P P$. Como $\left(\tilde{\mathrm{x}}_{1}, \tilde{\mathrm{x}}_{2}\right)$ é solução ótima de $P P$,

$$
T \mathrm{x}_{2 k-1}+T \mathrm{x}_{2 k} \leq T \tilde{\mathrm{x}}_{1}+T \tilde{\mathrm{x}}_{2}, k=1 . . \frac{L}{2}
$$

Somando as equações (B.2) e usando (B.1) obtém-se 


$$
\sum_{k=1}^{L} T \mathrm{x}_{k} \leq \frac{L}{2}\left[T \tilde{\mathrm{x}}_{1}+T \tilde{\mathrm{x}}_{2}\right] \stackrel{\text { (B.1) }}{=} \sum_{k=1}^{L} T \tilde{\mathrm{x}}_{k}
$$

Portanto, para $L \operatorname{par}\left(\tilde{\mathrm{x}}_{1}, \cdots, \tilde{\mathrm{x}}_{L}\right)$ é solução ótima para o problema $P R C$.

Seja $L$ ímpar e $\left(\hat{\mathrm{x}}_{1}, \hat{\mathrm{x}}_{2}\right)$ uma solução ótima de $P I$. A solução $\left(\hat{\mathrm{x}}_{1}, \cdots, \hat{\mathrm{x}}_{L}\right)$, em que $\hat{\mathrm{x}}_{k}=\hat{\mathrm{x}}_{1}$ para $k$ ímpar e $\hat{\mathrm{x}}_{k}=\hat{\mathrm{x}}_{2}$ para $k$ par é factível para o problema $P R C$ pois

$$
P \hat{\mathrm{x}}_{k}+P \hat{\mathrm{x}}_{k+1}=P \hat{\mathrm{x}}_{1}+P \hat{\mathrm{x}}_{2} \leq \mathbf{1}, k=1 . . L-1 .
$$

Para esta solução,

$$
T \hat{\mathrm{x}}_{1}+\cdots+T \hat{\mathrm{x}}_{L}=\frac{L+1}{2} T \hat{\mathrm{x}}_{1}+\frac{L-1}{2} T \hat{\mathrm{x}}_{2}
$$

Seja $\left(\mathrm{x}_{1}, \cdots, \mathrm{x}_{L}\right)$ uma solução factível para $P R C$. Como $P \mathrm{x}_{k}+P \mathrm{x}_{k+1} \leq \mathbf{1}, k=1 . . L-1$ então $\left(\mathrm{x}_{k}, \mathrm{x}_{k+1}\right), k=1 . . L-1$ são factíveis para $P I$. Como $\left(\hat{\mathrm{x}}_{1}, \hat{\mathrm{x}}_{2}\right)$ é solução ótima de $P I$, então

$$
\frac{L+1}{2} T \mathrm{x}_{k}+\frac{L-1}{2} T \mathrm{x}_{k+1} \leq \frac{L+1}{2} T \hat{\mathrm{x}}_{1}+\frac{L-1}{2} T \hat{\mathrm{x}}_{2}, k=1 . . L-1 .
$$

Logo, para $j=1 . . \frac{L-1}{2}$ segue que

$$
\begin{aligned}
\left(\frac{L+1}{2}-j\right)\left[\frac{L+1}{2} T \mathrm{x}_{2 j-1}+\frac{L-1}{2} T \mathrm{x}_{2 j}\right] & \leq\left(\frac{L+1}{2}-j\right)\left[\frac{L+1}{2} T \hat{\mathrm{x}}_{1}+\frac{L-1}{2} T \hat{\mathrm{x}}_{2}\right] \\
j\left[\frac{L+1}{2} T \mathrm{x}_{2 j+1}+\frac{L-1}{2} T \mathrm{x}_{2 j}\right] & \leq j\left[\frac{L+1}{2} T \hat{\mathrm{x}}_{1}+\frac{L-1}{2} T \hat{\mathrm{x}}_{2}\right]
\end{aligned}
$$

Somando as equações (B.4) e (B.5), para $j=1 . . \frac{L-1}{2}$ e por (B.3), obtém-se

$$
\left(\frac{L^{2}-1}{4}\right) \sum_{k=1}^{L} T \mathrm{x}_{k} \leq\left(\frac{L^{2}-1}{4}\right)\left[\frac{L+1}{2} T \hat{\mathrm{x}}_{1}+\frac{L-1}{2} T \hat{\mathrm{x}}_{2}\right] \stackrel{(\mathrm{B} .3)}{=}\left(\frac{L^{2}-1}{4}\right) \sum_{k=1}^{L} T \hat{\mathrm{x}}_{k}
$$

Portanto, para $L$ ímpar $\left(\hat{\mathrm{x}}_{1}, \cdots, \hat{\mathrm{x}}_{L}\right)$ é solução ótima para $P R C$. 